\title{
Diversification of the C-TERMINALLY ENCODED PEPTIDE (CEP) gene family in angiosperms, and evolution of plant-family specific CEP genes
}

\author{
Huw A Ogilvie*, Nijat Imin and Michael A Djordjevic
}

\begin{abstract}
Background: Small, secreted signaling peptides work in parallel with phytohormones to control important aspects of plant growth and development. Genes from the C-TERMINALLY ENCODED PEPTIDE (CEP) family produce such peptides which negatively regulate plant growth, especially under stress, and affect other important developmental processes. To illuminate how the CEP gene family has evolved within the plant kingdom, including its emergence, diversification and variation between lineages, a comprehensive survey was undertaken to identify and characterize CEP genes in 106 plant genomes.

Results: Using a motif-based system developed for this study to identify canonical CEP peptide domains, a total of 916 CEP genes and 1,223 CEP domains were found in angiosperms and for the first time in gymnosperms. This defines a narrow band for the emergence of CEP genes in plants, from the divergence of lycophytes to the angiosperm/ gymnosperm split. Both CEP genes and domains were found to have diversified in angiosperms, particularly in the Poaceae and Solanaceae plant families. Multispecies orthologous relationships were determined for $22 \%$ of identified CEP genes, and further analysis of those groups found selective constraints upon residues within the CEP peptide and within the previously little-characterized variable region. An examination of public Oryza sativa RNA-Seq datasets revealed an expression pattern that links OsCEP5 and OsCEP6 to panicle development and flowering, and CEP gene trees reveal these emerged from a duplication event associated with the Poaceae plant family.

Conclusions: The characterization of the plant-family specific CEP genes OSCEP5 and OsCEP6, the association of CEP genes with angiosperm-specific development processes like panicle development, and the diversification of CEP genes in angiosperms provides further support for the hypothesis that CEP genes have been integral to the evolution of novel traits within the angiosperm lineage. Beyond these findings, the comprehensive set of CEP genes and their properties reported here will be a resource for future research on CEP genes and peptides.
\end{abstract}

Keywords: C-terminally encoded peptide, Gene family, Signaling peptides, GC-biased gene conversion, Panicle development, Orthology detection, Angiosperm evolution

\section{Background}

The past decade has seen a paradigm shift in our understanding of plant growth and development. An important revelation has been the discovery of over 10 different multigene families that generate small secreted peptide signals as their mature biologically active products (hereafter referred to as signaling peptides). It is now recognized that these peptides work in parallel with

\footnotetext{
* Correspondence: huw.ogilvie@anu.edu.au

Research School of Biology, The Australian National University, Canberra ACT 0200, Australia
}

\section{Biomed Central

phytohormones to control important aspects of plant growth and development [1-4]. Expression studies show that genes coding for signaling peptides are expressed in discrete locations [5], where the resulting peptides non-cell autonomously regulate biological and physiological processes which enable plants to develop and adapt to environmental changes $[1,6,7]$. Signaling peptides regulate processes of fundamental importance including cell proliferation and expansion, meristem maintenance, gravitropism, pollen guidance, fertilization, abscission, and the development of stomata, vascular tissues, root hairs, lateral roots and root nodules [1,2,4,6-10]. 
One of these families is the C-TERMINALLY ENCODED PEPTIDE (CEP) gene family. CEP genes encode an $N$-terminal secretion signal (NSS), a variable domain, one or more CEP domains and a short $C$-terminal extension [11]. The 162 CEP genes discovered so far are single exon genes [6], and structure-activity studies suggest that the CEP domain is excised from the CEP prepropeptide to become a 15 amino acid (AA), post-translationally modified peptide $[7,11]$.

Several studies indicate that CEP peptides regulate plant root and shoot growth, and affect lateral root and root nodule development $[6,7,9,11]$. Recently, a knockout of AtCEP3 confirmed its role as a negative regulator of root development in response to abiotic stresses [6]. The paucity of CEP mutants has hampered detailed analyses of function, but overexpression and reporter gene studies suggest CEP peptides play important roles in a wide variety of processes in plants beyond controlling root growth and nodule development $[6,7,9,11]$.

Preliminary phylogenetic studies $[6,7,9]$ identified many CEP genes in angiosperms and root-knot nematodes (RKN) with CEP domains similar to those from the original five CEP genes discovered in Arabidopsis thaliana [11]. Unlike the CLAVATA3/EMBRYO SURROUNDING REGION (CLE) multigene family, CEP genes were absent in the earliest diverging lineages of plants or in other nematodes (including the closely related false-RKN and the more distantly related cyst nematodes) $[6,9,12,13]$. Those early studies also identified genes in angiosperms with domains more distant from the original CEP domains, termed "group II CEPs", and in gymnosperms, termed "CEP-likes" $[6,9]$. We term CEP genes which are structurally similar the originally discovered CEP genes "canonical CEPs". For a gene to be classified as a canonical CEP, it must have an NSS, variable region, and a CEP domain close in sequence to the originally discovered CEP domains (i.e., not group II or CEP-like).

Previous studies of CEP genes in angiosperms used BLAST to identify CEPs in a haphazard way using both genomic and expressed sequence tag resources [6,9]. In addition, it was not clear if canonical CEP genes are present in gymnosperms or to what extent CEP genes have diverged between plant families. The lack of a comprehensive survey of CEP genes across the plant kingdom has precluded an analysis of sequence diversity, orthology and selection pressure within this gene family. Recently, new and better methods of detecting small peptide encoding genes have been developed, based on motif identification and the prediction of one and two exon gene models [14].

Here, we have developed a motif identification system to detect CEP genes. An iteratively generated positionspecific probability matrix (PSPM) was used to comprehensively identify canonical CEP genes using high quality genomic information from a broad range of plants. The diversification of CEP gene and CEP domain sequences was characterized by calculating intra-organism pairwise distances (IOPDs). CEP gene orthology was determined using an extended Reciprocal Best Hit ( $\mathrm{RBH}$ ) method capable of detecting orthologous genes in multiple species. The comprehensive identification of CEP genes and orthologous groups enabled selection pressure on residues along the entire CEP prepropeptide to be identified and quantified using ratios of non-synonymous to synonymous substitutions. The influence of GC bias on CEP peptide AA distribution was determined. For the first time, canonical CEP genes were identified in gymnosperms; this defines a new limit on the latest possible emergence of CEP genes. Finally, CEP gene expression in Oryza sativa was examined using publicly available RNA-Seq datasets to reveal a developmental transition in expression of CEP genes from OsCEP6 in the booting panicle to OsCEP5 in the flowering panicle, strongly suggesting these genes play a role in panicle development.

\section{Results}

\section{Canonical CEP genes identified in gymnosperms and angiosperms}

To identify canonical CEP genes, 106 plant genome assemblies (Additional file 1: Table S1) spanning 80 genera and 39 families across the plant kingdom were scanned for open reading frames (ORF) with an NSS and one or more canonical CEP domains. Using a PSPM iteratively generated from previously identified CEP domains (Additional file 2), 916 CEP genes and 1,223 CEP domains were identified across seed plants (Table 1). Previously unknown CEP genes were identified using this method even in well-studied model organisms, including two new genes in Medicago truncatula, MtCEP12

Table 1 Summary of canonical CEP sequences identified in gymnosperms, angiosperms, and selected families within those clades

\begin{tabular}{llll}
\hline & CEP genes & CEP domains & Domains per gene \\
\hline Seed plants* & $\mathbf{9 1 6}$ & $\mathbf{1 , 2 2 3}$ & $\mathbf{1 . 3 4}$ \\
Angiosperms** $^{*}$ & $\mathbf{8 6 0}$ & $\mathbf{1 , 1 6 7}$ & $\mathbf{1 . 3 6}$ \\
Brassicaceae & 102 & 166 & 1.63 \\
Fabaceae & 124 & 149 & 1.20 \\
Poaceae & 86 & 95 & 1.10 \\
Rosaceae & 61 & 72 & 1.18 \\
Solanaceae & 70 & 70 & 1.00 \\
Gymnosperms*** & $\mathbf{5 6}$ & $\mathbf{5 6}$ & $\mathbf{1 . 0 0}$ \\
Pinaceae & 55 & 55 & 1.00
\end{tabular}

*Seed plants include all angiosperms and gymnosperms.

**Angiosperms include Brassicaceae, Fabaceae, Poaceae, Rosaceae, Solanaceae and unlisted angiosperm families.

***Gymnosperms include Pinaceae and unlisted gymnosperm families. 
and MtCEP13, and two new genes in O. sativa, OsCEP6 and OsCEP7 (Additional file 1: Table S2).

Out of the 12 canonical CEP genes and three "group II" CEP genes previously identified in $A$. thaliana $[6,9]$, all 12 canonical genes were re-identified and all three group II genes were rejected using this method (Additional file 1: Table S2). This result supports the sensitivity and specificity of our method for identifying CEP genes, and reinforces a previous finding [9] that group II CEP genes are phylogenetically distinct from canonical CEP genes.

Our data confirmed the absence of CEP genes $[6,9]$ from the genome assemblies of the bryophyte moss Physcomitrella patens [15] or the lycophyte Selaginella moellendorffii [16]. 860 CEP genes were identified in angiosperms (Table 1), including 13 in the genome assembly of the basal angiosperm Amborella trichopoda [17] (Additional file 1: Table S2). For the first time, 56 canonical CEP genes were identified in gymnosperm genomes. These included 55 CEP genes from the conifer family Pinaceae (Table 1) and one CEP gene from a gymnosperm outside the Pinaceae family, in the low coverage genome assembly [18] of Gnetum gnemon (Additional file 1: Table S2).

Many CEP domain sequences were shared by multiple genes across species; there were only 485 unique CEP domain sequences out of 1,223 total CEP domains identified. While the most common number of genes per domain sequence was one, 190 domain sequences were found in at least two genes and the most prevalent domain sequence (DFRPTAPGHSPGVGH) was identified in 53 different genes across the rosid clade of eudicots (Figure 1, Additional file 1: Table S3).

Notably, out of the CEP domains identified in the genome of the Pinaceae species Pinus taeda [19], two had an identical AA sequence (AFRPTSSGHSPGVGH) to a CEP domain identified in the genome of the angiosperm Kalanchoe fedtschenkoi [20]. The similarity of any given sequence to a PSPM can be quantified using a bit score, and therefore the bit score of a CEP domain compared to the canonical CEP PSPM is a measure of how CEP-like that domain is. By this measure, the domain sequence shared by $P$. taeda and K. fedtschenkoi was equal 19th most CEP-like out of the 485 unique CEP domain sequences (Additional file 1: Table S3). This analysis clearly demonstrates that canonical CEP genes are present in gymnosperms.

While all CEP genes identified in this study included a variable region, consistent with the five original CEP genes reported in $A$. thaliana [11], 53 CEP genes (representing $5.8 \%$ of all CEP genes identified in seed plants) were identified which lacked $C$-terminal extensions (Additional file 1: Table S2). These genes have a stop codon immediately after the $C$-terminal end of the

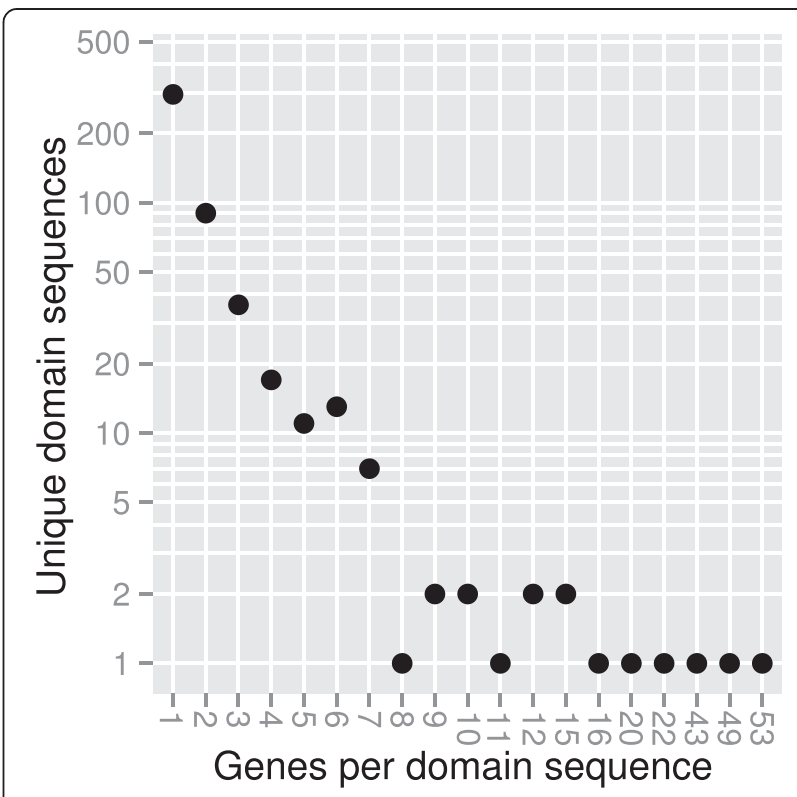

Figure 1 The distribution of the number of genes associated with each unique domain sequence. While 295 CEP domain sequences are present in only one CEP gene, 190 were present in multiple (sometimes many) CEP genes.

15 AA CEP domain, which defines the CEP domain's right border.

\section{Diversification of CEP gene and domain sequences}

To measure the diversity of CEP gene and CEP domain sequences, and the difference in sequence diversity between plant families, intra-organism pairwise distances (IOPD) were calculated. IOPDs are the genetic distances between all pairs of CEP genes and CEP domains identified in the genome assembly of an individual organism. For the families best represented among genome assemblies - the gymnosperm family Pinaceae, and the angiosperm families Brassicaceae, Fabaceae, Poaceae, Rosaceae and Solanaceae - IOPD distributions were generated by aggregating IOPDs by plant family.

For both CEP genes and CEP domains, all five wellrepresented angiosperm families featured significantly $(P<0.001)$ more sequence diversity than Pinaceae. Intriguingly, the CEP gene and CEP domain sequence diversity of Solanaceae (within the eudicot and asterid clades) and Poaceae (within the monocot clade) were both significantly $(P<0.05)$ greater than Brassicaceae, Fabaceae or Rosaceae (Figure 2). Solanaceae and Poaceae do not share a common ancestor to the exclusion of the other well-represented families [21], so this pattern must require either independent increases in CEP sequence diversity in the Solanaceae and Poaceae lineages, or a loss of sequence diversity in the rosid clade which includes the other well-represented angiosperm plant families. 


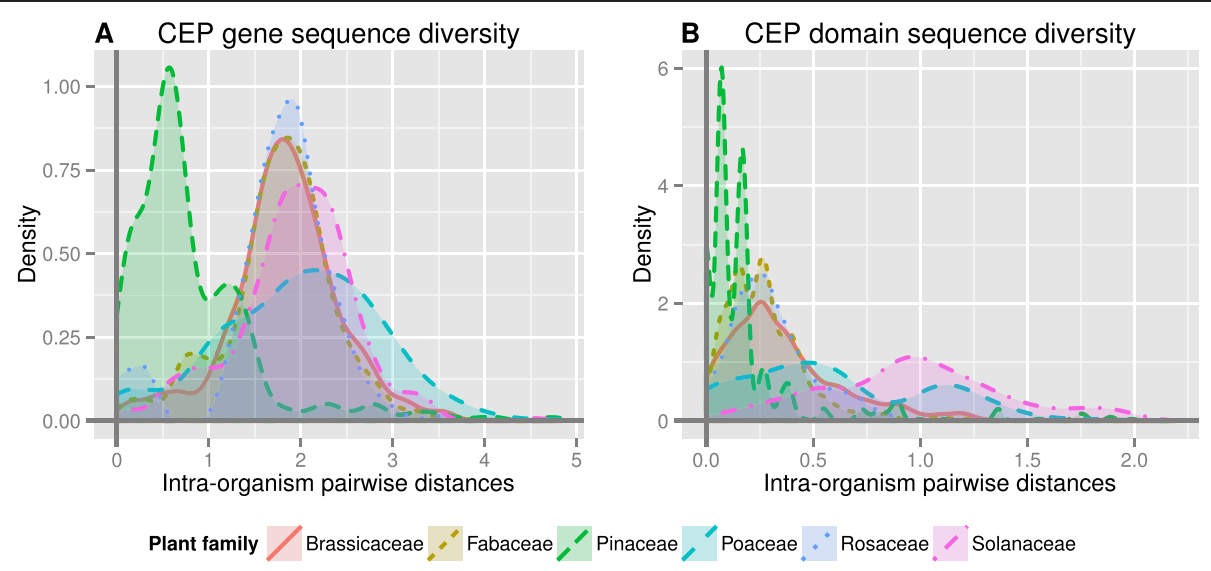

Figure 2 A comparison of CEP gene (A) and CEP domain (B) sequence diversity between seed plant families. These density plots of sequence diversity graph the distributions of pairwise genetic distances of CEP genes and domains within a single organism, aggregated by plant family. Genetic distances were calculated based on the AA sequences, using a maximum likelihood estimation. Tukey's test reveals that both CEP gene and domain sequence diversity is significantly greater in all angiosperm families than in the gymnosperm family Pinaceae $(P<0.001)$. Additionally, the CEP gene and domain sequence diversity of Poaceae and Solanaceae are significantly $(P<0.05)$ greater than in other angiosperm families.

Patterns of conservation within the CEP domain are apparent when comparing PSPMs generated separately for each plant family - particularly the $C$-terminal residues at positions 7 through 15 (most commonly [PS]GHSPG[VI] $\mathrm{GH}$ ) which are usually conserved within and between plant families (Figure 3). In contrast, the $N$-terminal residues from position 1 to 5 are notably more variable within Poaceae and Solanaceae CEP domains than in other families (Figure 3D and F), explaining the increased diversity seen in CEP domain IOPDs for Poaceae and Solanaceae. For example, in other families the residue at position 2 is usually phenylalanine, but can be other AAs in Solanaceae (Figure 3F), and is never phenylalanine in Poaceae (Figure 3D).

In addition, while position 6 shows a high degree of variability in all angiosperm plant families we analyzed, it is usually either serine or alanine in Pinaceae (Figure 3C). Another notable difference between angiosperm and gymnosperm CEP domains is at position 7, which is highly conserved as proline in angiosperm plant families (Figure 3A, B, D, E and F) but in Pinaceae (Figure 3C) is always serine. Finally, diversity in chemical properties is not necessarily correlated with diversity of AAs. For example, while the AA distribution at position 13 varies by plant family, three of the most common AAs at that position - isoleucine, valine and methionine - are all hydrophobic.

\section{Selective constraints on residues within and outside CEP domains}

Orthologous groups of CEP genes were identified using the $\mathrm{RBH}$ method, extended to more than two organisms

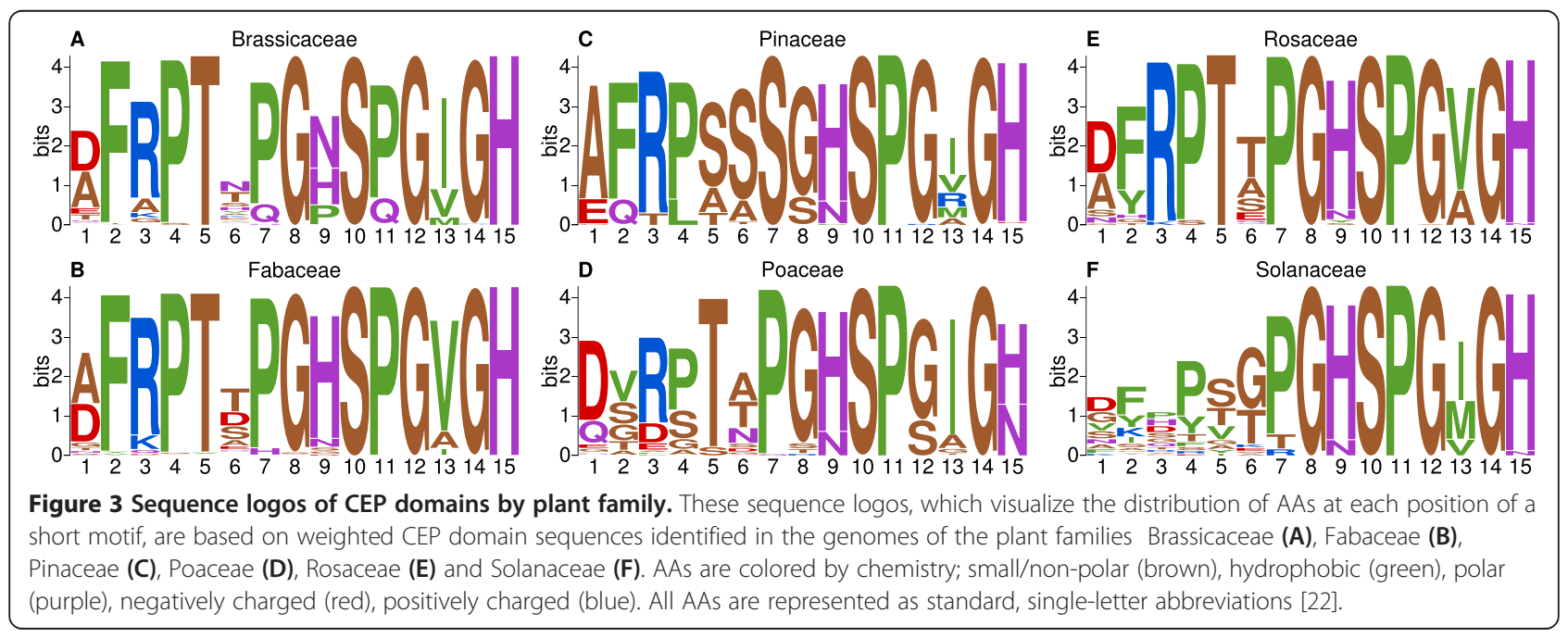


(see Methods). In angiosperms, 34 orthologous groups were identified. With one exception, all groups were restricted to species within a single plant family: either Brassicaceae, Cucurbitaceae, Fabaceae, Poaceae, Rosaceae or Solanaceae. Orthologous group 10 included genes from Brassicaceae as well as from the genome assembly of Tarenaya hassleriana [23], a species from the sister family to Brassicaceae, Cleomaceae. No orthologous groups were identified in gymnosperms. As only three high coverage gymnosperm genome assemblies $-P$. taeda [19], Picea abies [18] and Picea glauca [24] - are available, this may be due to the minimum requirement of four orthologs per group imposed in this study. In total, 202 CEP genes were classified into orthologous groups, $22 \%$ of all CEP genes identified in this study.

To identify any residues in the CEP prepropeptide that are under negative selection, ratios of non-synonymous to synonymous substitutions $\left(\mathrm{d}_{\mathrm{N}} / \mathrm{d}_{\mathrm{S}}\right)$ were estimated for all residues of all orthologous groups (Additional file 3). Ratios below 1 indicate a selective constraint at that position, favoring specific AAs [25]. One exemplary orthologous group was selected from each well-represented angiosperm plant family. Significantly constrained residues were found across the CEP coding region in each exemplary group, including within the NSS (light red), variable region (grey), CEP domain (blue) and $C$-terminal extension (green) (Figure 4).

Although conserved residues were more common within the CEP domain than outside of it, short conserved motifs were identified in variable regions. These include the $\mathrm{Cx}_{2} \mathrm{C}$ motif present in orthologous groups 3 and 4 (Figure $4 \mathrm{~A}$ and $\mathrm{B}$ ). Combined with the $\mathrm{Hx}_{5} \mathrm{H}$ motif present within the CEP domains, this is reminiscent of a $\mathrm{Cys}_{2} \mathrm{His}_{2}$ zinc finger (ZnF) domain, but with a considerably longer gap between the second cysteine and first histidine than the 12 amino acid gaps seen in DNA and protein-binding $\mathrm{Cys}_{2} \mathrm{His}_{2}$ domains [26]. Orthologous group 8 includes a polyproline region (Figure 4C). Orthologous group 28 (Figure 4E) includes a cluster of conserved residues with the motif TxSPD in the variable region, which is potentially a binding site for posttranslational modification by proline-directed kinases [27].

Consistent with the increased variation observed at position 2 of the CEP domain in Poaceae and Solanaceae, the highest probability density (HPD) interval for the $d_{N} / d_{S}$ ratio at that position of orthologous group 8 (from Solanaceae; Figure 4C) was 0.52 to 4.1, and for orthologous group 28 (from Poaceae; Figure 4E) was 0.42 to 1.97 . As these intervals include 1.0 , no evidence of a selective constraint at that position was observed for either group. However, the upper HPD limits for the $\mathrm{d}_{\mathrm{N}} / \mathrm{d}_{\mathrm{S}}$ ratio at position 2 of orthologous groups 3 (from Rosaceae; Figure 4A), 4 (from Fabaceae; Figure 4B) and 10 (from Brassicaceae and Cleomaceae; Figure 4D) were
$0.99,1.03$ and 0.76 respectively. Since these limits are below 1 for groups 3 and 10, this is evidence of a selective constraint for phenylalanine at that position, which for group 4 is marginally non-significant. Despite the different AAs present at position 13 in different orthologous groups, a significant selective constraint was observed at all groups except group 8 (Figure 4).

\section{The relationship between GC content and CEP domain residues by plant family}

To explore a potential basis for the different AA frequencies observed within the CEP domain for different plant families, the proportion of guanine and cytosine nucleotides (GC content) was calculated for all CEP genes identified in this study. Furthermore, the distribution of CEP gene GC content in the six well-represented plant families was calculated (Figure 5), and pairwise distances in GC content between those families statistically tested. The GC content of Poaceae CEP genes was significantly higher $(P<0.001)$ than any other plant family, and the GC content of Solanaceae CEP genes was significantly lower than any other plant family $(P<0.001)$.

The distributions of AAs at position 2 of the Poaceae and Solanaceae CEP domains (Figure 6) is consistent with the stark difference in GC content between CEP genes from those plant families. The most common AAs at this position in Poaceae - valine, serine, glycine and threonine - can be encoded using GC rich codons containing two or three guanine or cytosine nucleotides (Figure 6D). In contrast to this, the most common AAs at this position in Solanaceae - phenylalanine, tyrosine, lysine and isoleucine - can only be encoded using GC poor codons containing zero or one guanine or cytosine nucleotides (Figure 6F). However in position 13, where a selective constraint was often observed (Figure 4), the most common AA for both Poaceae and Solanaceae CEP domains was isoleucine, which utilizes GC poor codons (Figure 6D and Figure 6F).

\section{Expression analysis of the complete set of Oryza sativa CEP genes}

Given that CEP gene orthology was limited to single plant families, or at most sister plant families, this suggests some CEP genes could be phylogenetically and functionally unique to specific plant families. To investigate this possibility using a well-studied model organism, public RNA-Seq datasets were reanalyzed to measure the expression of all CEP genes identified in the Poaceae species O. sativa. A key feature of the Poaceae family is the development of grain-type seeds, and in Oryza these develop on characteristic loose panicles. Independent studies have been conducted using RNA-Seq to investigate the transcriptomes of different tissues of the two O. sativa subspecies, Indica [28] and Japonica [29]. 


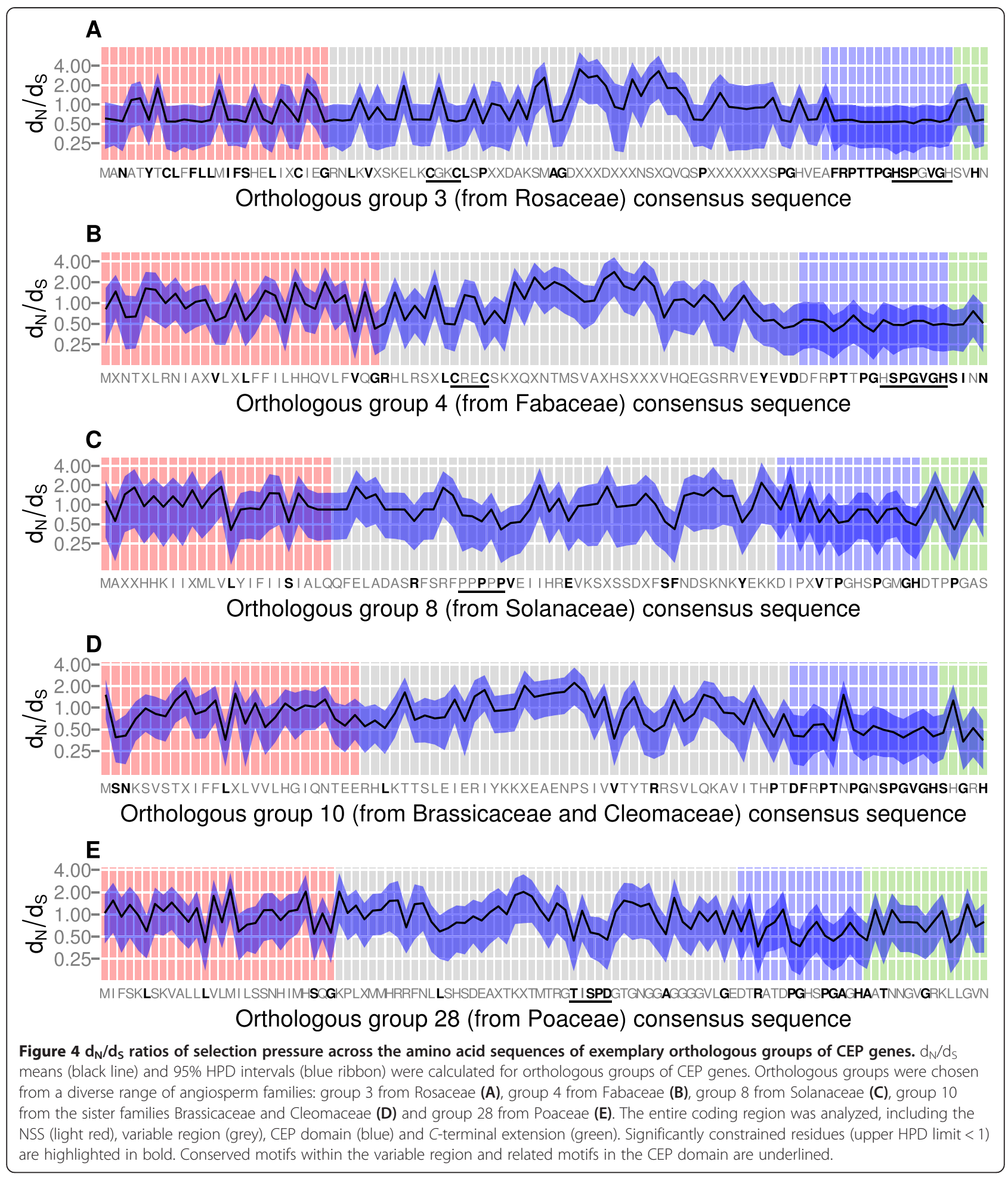

The reanalysis uncovered a distinctive pattern of CEP gene expression in both subspecies, where OsCEP6 is predominantly expressed in the (earlier) booting panicle, and OsCEP5 is predominantly expressed in the (later) flowering panicle (Figure 7). This expression pattern implies a possible role for those CEP genes in panicle development.
Kingdom-wide phylogenetic trees of the CEP gene family To infer when OsCEP5 and OsCEP6 emerged in the evolutionary history of plants, maximum likelihood phylogenetic genes of all CEP genes identified in this study were reconstructed based on both AA and nucleotide sequences. In both AA and nucleotide trees, OsCEP5 


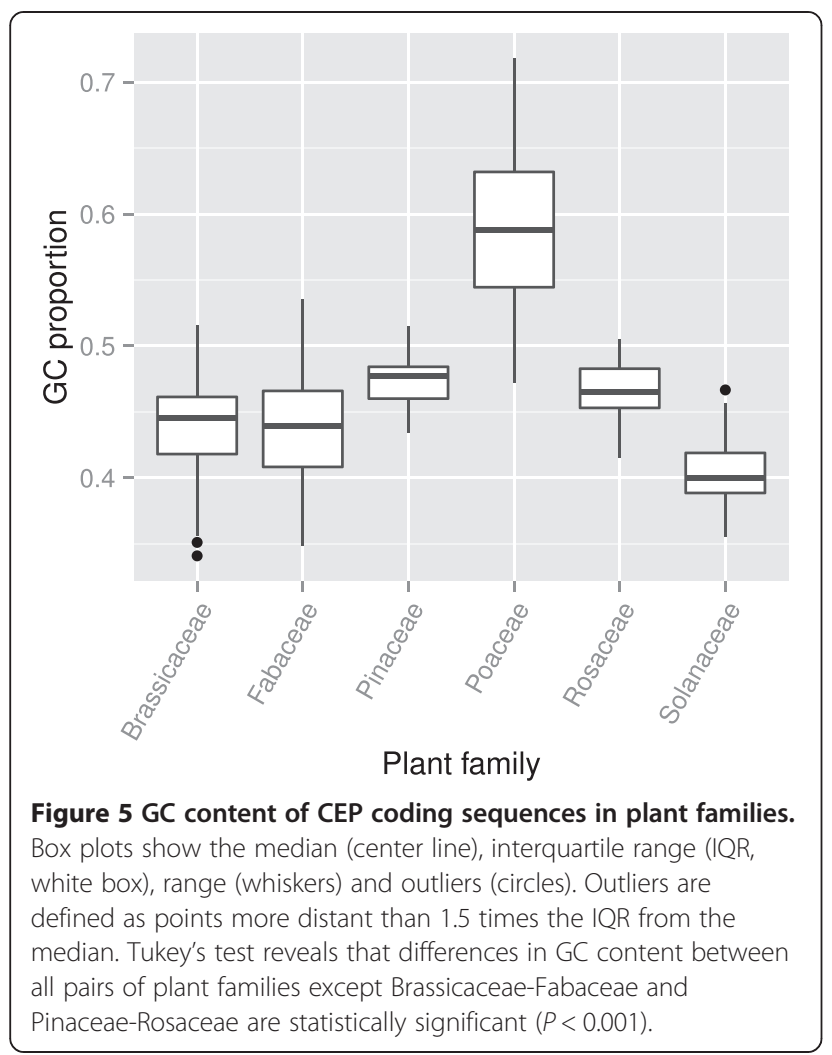

and OsCEP6 were located within a single cluster of Poaceae CEP genes (Figure 8). Bootstrap support values were calculated for both trees, and in the AA tree the cluster has a strong support value of 91 (Additional file 4), while in the nucleotide tree it has a weak support value of 49 (Additional file 5).

A closer inspection revealed that OsCEP5 and OsCEP6 were placed into separate lineages within each cluster, and each lineage includes CEP genes from a broad range of Poaceae species (Figure 9). This is indicative of a gene duplication event having occurred prior to the evolution of Poaceae, or early in the Poaceae lineage. This identification of OsCEP5 and OsCEP6 as sister paralogs, and their conservation throughout Poaceae, suggests they may play a role in inflorescence development in other Poaceae species besides $O$. sativa.

Relationships between clusters of CEP genes from different plant families are difficult to resolve due to low bootstrap support values (Additional files 4 and 5). This may be due to the short length of the CEP coding sequence, leading to an insufficient number of phylogenetically informative residues to resolve the deep relationships between plant-family specific clusters. This is highlighted by the shortest CEP coding sequence identified, which is from the genome assembly of Azadirachta indica [30]. Its CEP coding sequence is 48 AAs in length, including an NSS of 19 AAs, a variable region of 10 AAs, a CEP domain of $15 \mathrm{AAs}$ and a $C$-terminal extension of 4 AAs (Additional file 1: Table S2).

\section{Discussion}

Our method identified canonical CEP genes across angiosperms and gymnosperms, placing an earlier limit on CEP gene emergence

Previous studies have used BLAST to identify CEP genes in a haphazard way across existing sequence databases $[6,9]$. By developing a systematic approach where essential features of a CEP gene (the NSS and CEP domain) are scanned for in all ORFs, 916 CEP genes were identified, greater than five-fold the number of genes identified in a previous study [6]. This includes genes from species where canonical CEP genes have not been previously identified, most importantly gymnosperms including $G$. gnemon and Pinaceae species, demonstrating that CEP genes are not limited to angiosperms but are present across seed plants.

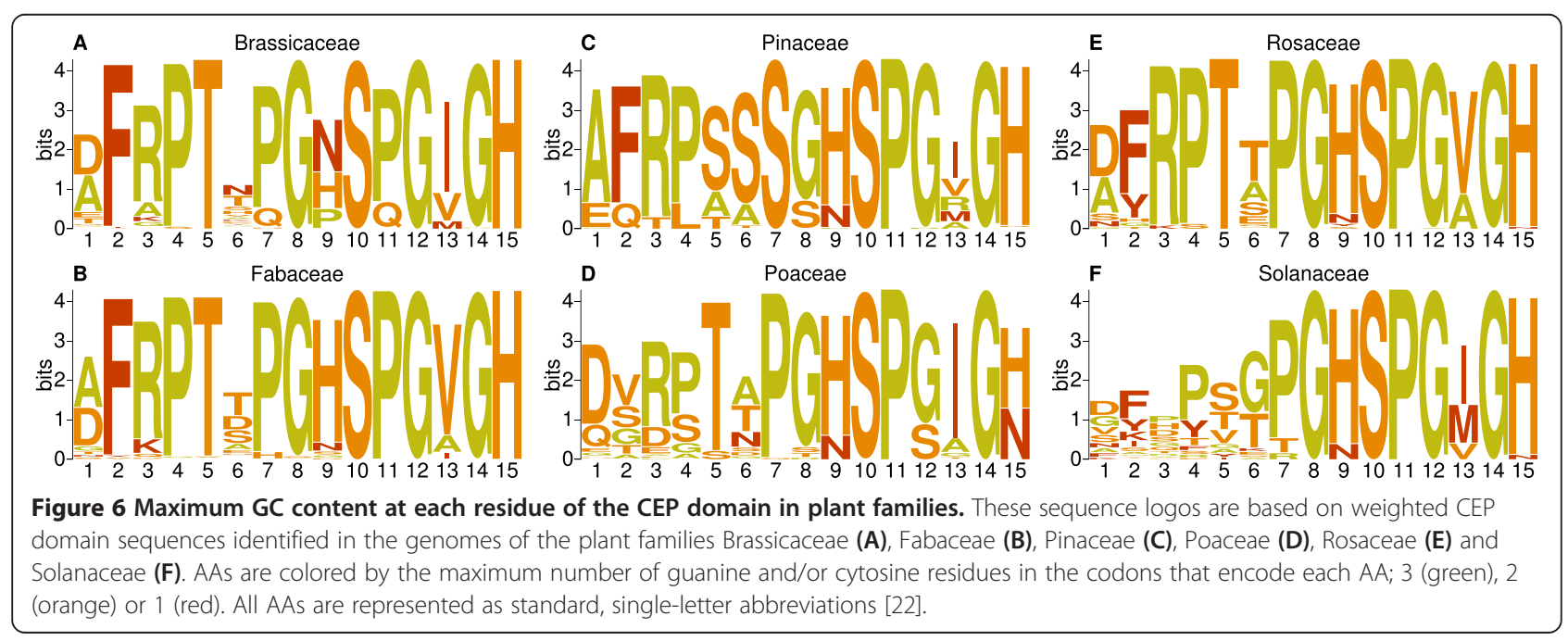




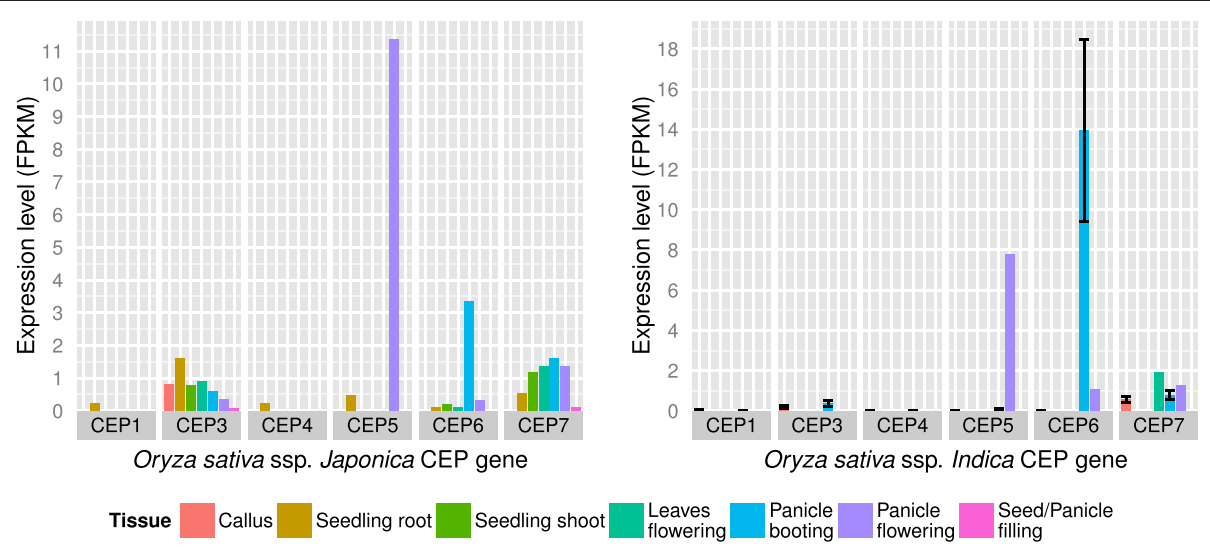

Figure 7 CEP gene expression during Oryza sativa development, replicated in Japonica and Indica subspecies. Alternating high expression of OsCEP6 during panicle booting, then OsCEP5 during panicle flowering, is consistent across subspecies. Error bars indicate standard error for tissues with replicates. $\mathrm{N}=15$ for Indica booting panicle, $\mathrm{N}=14$ for Indica callus, otherwise $\mathrm{N}=1$.

The absence of CEP genes from the bryophyte moss $P$. patens or the lycophyte $S$. moellendorffii places a limit on earliest emergence of CEP genes after the divergence of those lineages, which is later than CLE genes which are present in both plants [31]. The positive identification of CEP genes in gymnosperms in this study places a limit on the latest emergence of CEP genes at the point of angiosperm/gymnosperm divergence. Further research is needed to determine whether CEP genes are present in monilophytes, which emerged after lycophytes but before the angiosperm/gymnosperm divergence [21]. The methods used in this study can be applied to monilophyte genome assemblies when they become available.

Additional CEP genes were also identified in previously analyzed species, for example the OsCEP6 gene in $O$. sativa, as well as additional CEP genes in the model legume M. truncatula. The comprehensive database of CEP genes and domains identified in this study (Additional file 1) will therefore be a resource to researchers working on CEP genes in both model and non-model organisms.

\section{CEP genes have diversified in angiosperms, especially in Solanaceae and Poaceae}

The comprehensive identification of CEP genes across a broad range of seed plants has enabled a partial elucidation of the evolutionary relationships between those genes. The newly identified gene OsCEP6 emerged from a gene duplication event in the Poaceae plant family, which also produced the previously identified gene OsCEP5. The expression pattern of these genes points to a role in panicle development, and their conservation within Poaceae points to a role throughout that family. These paralogs demonstrate that at least some CEP genes are plant family specific, rather than being conserved in sequence and function across seed plants or across angiosperms.
To an extent the evolution of plant-family specific genes parallels the evolution of the CLE gene family. Some CLE peptides present in the Poaceae species $O$. sativa lack close relatives in the Brassicaceae species $A$. thaliana [32], and three separate CLE genes in O. sativa regulate meristem maintenance in the shoot apical, inflorescence and floral meristems, instead of the single CLAVATA3 (CLV3) gene which serves that function in A. thaliana [33]. Multiple nodule-specific CLE genes have been identified in legumes which regulate nodulation in response to nitrate levels, a process specific to nodulating plants (mostly found in the Fabaceae family) [34].

However, the poor sequence diversity of CEP genes and CEP domains in gymnosperms differs from the CLE gene family, which has been found to be as diverse in the Pinophyta clade of gymnosperms as it is in angiosperms [35]. The lack of broadly conserved CEP domain sequences shared by gymnosperms and angiosperms differs from CLE peptides, many of which are closely conserved between Pinophyta (including Pinaceae) gymnosperms and the angiosperm $A$. thaliana. One important CLE peptide, TRACHEARY ELEMENT DIFFERENTIATION INHIBITORY FACTOR (TDIF), is perfectly conserved in sequence between Pinophyta and many angiosperm species including $A$. thaliana $[35,36]$.

This diversification of CEP genes and peptides in angiosperms (and the further diversification observed within Solanaceae and Poaceae), and the association of CEP genes with angiosperm-specific development, suggests that CEPs have been integral to the evolution of novel traits within the angiosperm lineage. Apart from the emergence of the Poaceae-specific CEP genes OsCEP5 and OsCEP6 and their association with panicle development in O. sativa, CEP genes have also been implicated in legume nodule development [7] and in floral development [9], all angiosperm-specific processes. 


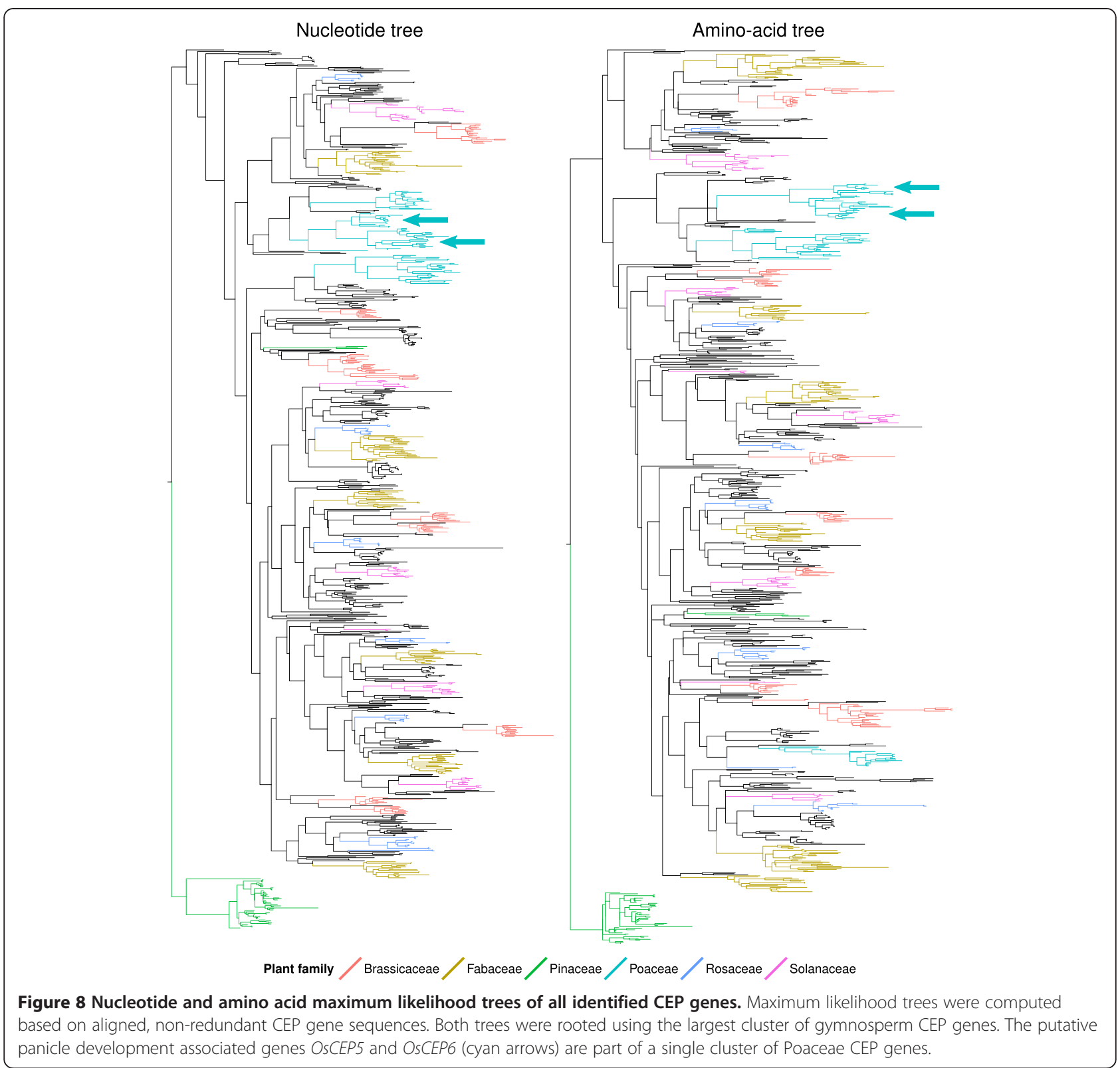

\section{Selective constraints on CEP residues support functional differences deriving from differences in amino acid sequence}

Identification of orthologous groups of CEP genes and the characterization of selection pressure within the CEP domains of those groups has revealed key drivers of AA distributions and increased CEP domain sequence diversity. In orthologous groups from plant families that exhibit the highest CEP gene and CEP domain sequence diversity, Poaceae and Solanaceae, no evidence for selective constraint was observed at position 2 of the CEP domain, consistent with the greater variability observed at that residue and at positions $1-5$ generally in those families.
Interestingly, AA distributions at position 2 differ between Poaceae and Solanaceae. In the absence of selective constraint, GC-biased gene conversion may alter the AA distribution at CEP peptide sites in Poaceae, by selecting for codons with higher GC content. GC-biased gene conversion is stronger in Poaceae than in other plant families [37], and the high GC content of Poaceae CEP genes reflects this phenomenon.

Across plant families, selective constraint was observed at position 13 of the CEP domain, which is typically a hydrophobic residue. However the exact residue varies between orthologous groups, suggesting that differences in CEP peptide sequences at this residue may be important to function. 


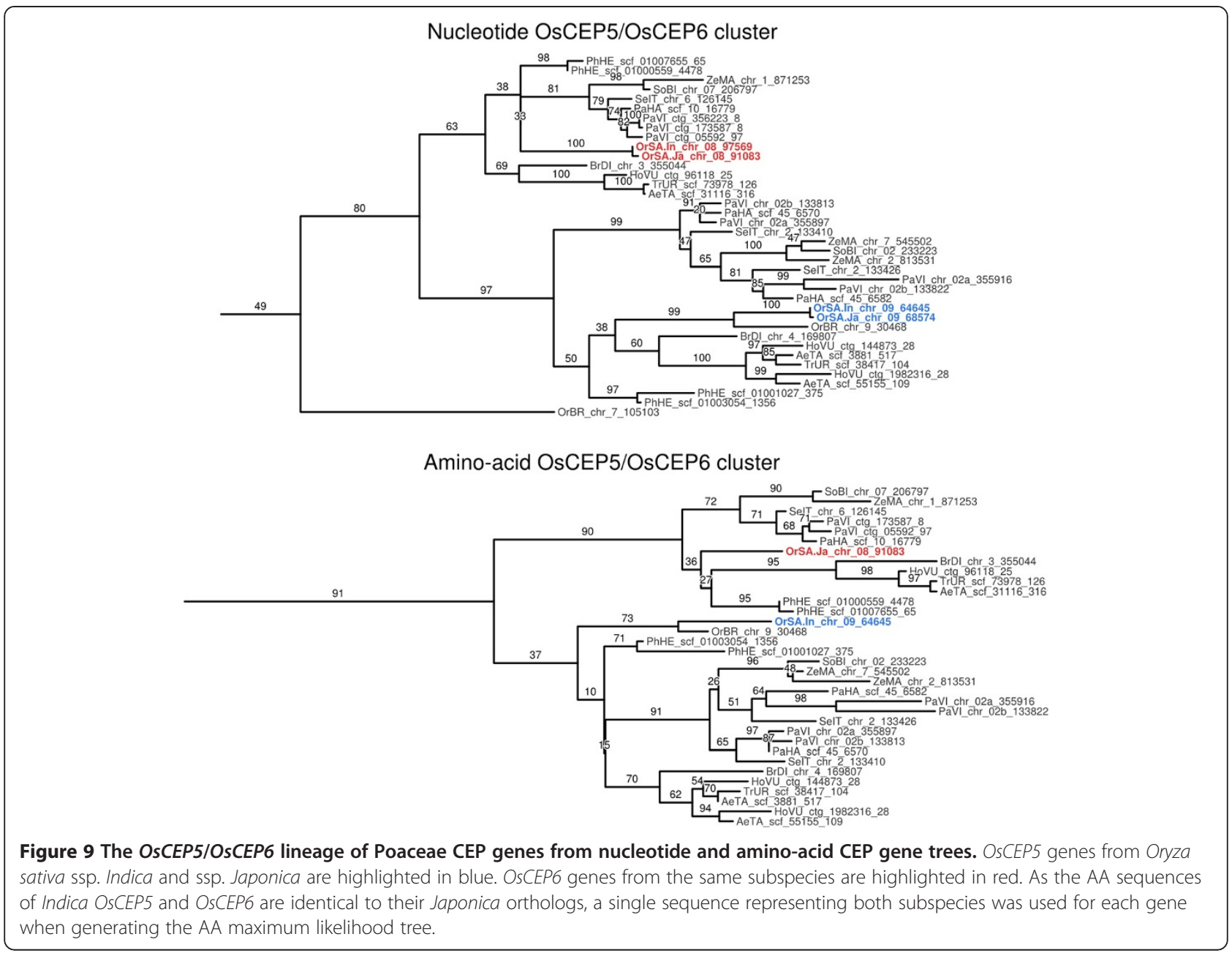

The presence of conserved motifs within the variable regions of orthologous groups suggests that those regions may also have a functional role beyond simply linking the NSS and CEP peptide domain. Biochemical investigation is needed to determine the function of conserved motifs observed within the variable region, including their influence on protein folding, post-translational modification and binding properties.

Recently a secreted kinase was identified which is responsible for the phosphorylation of proteins targeted to the ER-dependent secretion pathway [38], and therefore potentially phosphorylated conserved motifs within variable regions could be sites targeted during secretion for regulation or processing of the CEP prepropeptide. Another avenue of investigation following the results of this study is whether the $\mathrm{Cys}_{2} \mathrm{His}_{2}$-like motifs identified in orthologous groups 3 and 4 bind ions in the same way as $\mathrm{ZnF}$ domains, despite the longer gap between the pair of cysteine and the pair of histidine residues in CEP genes relative to the gap in $\mathrm{ZnF}$ domains.

Homopolymeric proline repeats have been observed in the CLE gene OsCLE502, where they act as linkers between multiple CLE domains [39]. However, the polyproline sequence in CEP orthologous group 8 from Solanaceae is located in the variable region, and this orthologous group only encodes a single CEP domain. Regardless of whether homopolymeric repeats can be functional, the bulk of evidence suggests they can evolve neutrally [40].

Both within the CEP domain and within the variable region, selective constraints at sites which differ between orthologous groups could contribute to functional specificity deriving from the coding sequence of CEP genes, rather than their expression patterns alone. This is supported by a previous study that showed different CEP genes driven by a $35 \mathrm{~S}$ overexpression promoter produced different phenotypes in A. thaliana [6].

\section{Conclusions}

Until recently, identification of signaling peptide gene family members, including CEP genes, has been based on searching public sequence databases for homologous sequences. Now that many plant genome assemblies are available, we have systematically identified many canonical 
CEP genes by searching for their two essential domains (NSS and CEP) in the ORFs of those assemblies. This has confirmed the presence of CEP genes in gymnosperms, placing a new limit on the latest possible emergence of CEP genes.

By studying multiple aspects of the molecular evolution of CEP genes (orthology, selection pressure, IOPDs and gene trees), we can conclude that CEP genes have diversified in angiosperms, particularly in the Poaceae and Solanaceae plant families. We have also demonstrated that the OsCEP5 and OsCEP6 genes evolved with the Poaceae plant family, and are implicated in panicle development. Together with previous CEP reporter gene studies implicating CEP genes in other angiospermspecific processes, this strongly suggests that the diversification of CEP genes has contributed to the evolution of angiosperms.

Finally, our approach can be applied to the study of other signaling peptide gene families, to shed further light on the evolutionary aspects and putative functions of signaling peptides in plants.

\section{Methods}

\section{Generating position specific probability matrices}

PSPMs, which describe the AA distribution at each site of a motif, were generated using the motif discovery tool MEME [41]. An initial PSPM of the CEP peptide domain was generated from an unweighted set of all previously identified [6] canonical CEP peptide domains. An improved second iteration of the CEP PSPM was generated from a weighted set of all CEP domains identified in plant genomes using the first PSPM. The CEP domains of specific plant families were characterized by generating separate PSPMs for each family, based on weighted sets of all CEP domains identified in those plant families using the second CEP PSPM.

For weighted sets, CEP domains weights were adjusted so that the weight for each CEP domain within a single ORF, the sum of weights for each ORF within a single genome assembly, as well as the sum of weights for each genome assembly were equal. A second weighting was applied to correct for over- and under-represented lineages using a species tree (Additional file 6). Over-represented lineages will contain more internal nodes, so CEP domain weights were reduced by $20 \%$ for each internal node from the root of the tree to each CEP domain's associated organism.

The species tree used when weighting CEP domains was based on a previous study on the relationship between plant families [21], and previous studies on the relationship between species of gymnosperms [42], Brassicaceae and Cleomaceae [43], Cucurbitaceae [44], Euphorbiaceae [45], Fabaceae [46], Fragaria [47], Lamiales [48], Malpighiales [49], Nicotiana [50], Oryza [51], Poaceae
[52], Rosaceae [53], Solanaceae [54] and the TriticumAegilops complex [55].

\section{Identifying CEP genes and CEP domains in plant genome assemblies}

Pseudomolecules of the $M$. truncatula genome assembly version 4.0 [56] contained CEP genes absent from version 3.5.2 [57], but version 3.5.2 included unanchored BACs and unplaced contigs containing CEP genes absent from version 4.0. Through visual inspection of preliminarily identified $M$. truncatula CEP sequences, we verified that no CEP genes from version 4.0 pseudomolecules were present in version 3.5.2 unanchored BACs and unplaced contigs, and vice versa. In order to analyze the most complete set of $M$. truncatula CEP genes, a hybrid assembly was produced consisting of version 4.0 pseudomolecules and version 3.5.2 unanchored BACs and unplaced contigs. A second round of visual inspection of CEP sequences identified in the hybrid assembly confirmed that version 4.0 pseudomolecule CEPs and version 3.5.2 unanchored BAC/unplaced contig CEPs were mutually exclusive.

The M. truncatula hybrid genome and all other plant genome FASTA files were homogenized and concatenated using a custom Python script (Additional file 7). ORFs longer than 50 AAs between in-frame stop codons were extracted using the EMBOSS tool getorf [58]. This enabled the detection of very short CEP genes, including genes where the coding sequence (CDS) was less than 50 AAs in length, as long as the CDS was contained within a genomic sequence of at least $50 \mathrm{AA}$ in length uninterrupted by stop codons. Extracted ORFs were interleaved across 100 output files so that later analysis would fit in available RAM. CEP domains were identified within extracted ORFs using the motif scanning tool FIMO [59], with a conservative $P$-value cut-off of $6 \times 10^{-11}$.

CEP ORFs should contain an NSS which begins at the $N$-terminus. As a diverse range of plant genes utilizing start codons which typically code for leucine have been identified [60-63], the NSS might begin at any in-frame methionine or leucine codon before the CEP domain. To test for the presence of NSSs and to identify the start codon of each CEP ORF, the NSS identification tool SignalP 4.1 [64] was used to quantify the likelihood of an NSS at every possible start codon. ORFs where no start codon had a SignalP score above 0.400 were unlikely to contain an NSS and discarded. Otherwise, the possible start codon with the highest SignalP score was used to define the $5^{\prime}$ end and $N$-terminus of the nucleotide and AA sequence respectively.

\section{Calculating and comparing pairwise genetic distances}

The genetic distance between all possible pairs of CEP genes and domains identified was estimated using the maximum likelihood algorithm implemented by PAML 
and the JTT AA substitution matrix $[65,66]$. Global alignments of AA sequence pairs for use with PAML were generated using the L-INS-i algorithm implemented in MAFFT [67] and the JTT substitution matrix.

The diversity of CEP genes and domains within specific plant families (IOPDs) were calculated by aggregating the genetic distances between CEP genes and between CEP domains from the same genome assembly by plant family. Density plots of the distributions of aggregated pairwise distances were generated using ggplot2 [68]. To calculate statistical differences between those distributions, pairwise distances were first rank-transformed due to the multimodality, skewing and unequal variances observed in the density plots. Tukey's test was used to calculate multipletesting corrected $P$-values for all pairwise comparisons of plant families.

Identifying orthologous relationships between CEP genes Orthologous groups of CEP genes were identified by extending the RBH method of identifying orthologous genes shared by two species [69]. First, RBH pairs of CEP genes between all species were identified using BLASTP [70], and a graph assembled of those pairs. Second, RBH pairs from the lowest to highest BLAST bit scores were checked to ensure that both genes in the pair share the same set of orthologs. When another gene is orthologous to only one gene of the original pair, the two genes of the RBH pair may belong to different orthologous groups, and the edge connecting the pair was deleted. After an edge was deleted, this process was repeated from the second step until both genes from all remaining pairs shared identical sets of orthologs. By pruning edges connecting potentially non-orthologous $\mathrm{RBH}$ pairs, the graph was reduced to clusters of genes within which every gene is connected to every other gene. Clusters of more than four genes were considered orthologous groups and labelled sequentially.

This algorithm is a special case of a previously described clustering $\mathrm{RBH}$ (cRBH) algorithm, which left unspecified the order in which $\mathrm{RBH}$ pairs were compared with other genes, and therefore which edges are deleted to produce the reduced graph [71]. By repeatedly evaluating $\mathrm{RBH}$ pairs in ascending order of their BLAST bit scores, the method in this study is deterministic and reproducible.

\section{Calculating ratios of non-synonymous to synonymous substitutions $\left(d_{N} / d_{S}\right)$}

Conservation of residues in orthologous groups was identified using $d_{N} / d_{S}$ ratios of non-synonymous to synonymous substitutions. The renaissance counting feature of the Bayesian phylogenetics package BEAST [72] was used to estimate $d_{N} / d_{S}$ ratios and 95\% HPD intervals for each orthologous group of CEP genes. A multiple sequence alignment of each orthologous group was generated using the L-INS-i algorithm and the JTT substitution matrix.
Poorly aligned columns were excised using the gappyout algorithm implemented by TrimAl [73]. For all groups, parameters were estimated using an HKY substitution model [74] with empirical base frequencies, a chain length of $10^{8}$ steps and a $10 \%$ burn-in. Graphs of $d_{N} / d_{S}$ ratios, HPD intervals and consensus sequences (generated using the EMBOSS tool cons [58]) were again generated using ggplot2.

\section{Comparing the GC content of CEP genes in different plant families}

The number of guanine and cytosine nucleotides as a proportion of all nucleotides (GC content) was calculated for each CEP gene. For specific plant families, the distribution of GC content in all genes in each family was visualized as a boxplot using ggplot2. To calculate statistical differences between those distributions, a logit transformation was applied to GC content to change proportions (which are bounded by 0 to 1 ) into log-odds (which are unbounded). Tukey's test was used to calculate multiple-testing corrected $P$-values for all pairwise comparisons of plant families.

\section{Calculating differential expression of CEP genes}

To calculate the expression levels of CEP genes, RNA-Seq reads were remapped to their reference genomes using the short-read aligner SMALT [75] with default settings. Then, based on the CEP genes identified, general feature format (GFF) files were generated for each assembly which specified CEP mRNA regions as beginning $100 \mathrm{nu}-$ cleotides upstream of the start codon and 300 nucleotides downstream of the stop codon. The mapped reads and GFF files were used as input for the cuffdiff program from the Cufflinks package [76], to calculate CEP gene expression as fragments per kilobase of exon model per million reads (FPKM).

\section{Reconstructing the phylogenetic tree of the CEP gene family}

The phylogenetic history of the CEP gene family across all plants was reconstructed using a maximum likelihood approach. First, ambiguous characters were stripped from the sequences of CEP genes identified in this study. Multiple sequence alignments (MSA) of all nucleotide sequences were generated using L-INS-i, and all AA sequences were aligned using L-INS-i and the JTT substitution matrix. Poorly aligned columns from both nucleotide and AA MSAs were excised using the gappyout algorithm implemented by TrimAl. 100 bootstrap replicates and maximum parsimony starting trees of the original and bootstrap sequence alignments were generated using the RAxML phylogenetic software [77]. Unrooted gene trees were reconstructed for the original MSA and each bootstrap replicate using the phylogenetic software ExaML 
[78]. The nucleotide and AA maximum likelihood trees were generated based on the general time reversible and JTT substitution models respectively. Both were generated using the CAT model of rate heterogeneity [79]. Finally, bootstrap support values were added to the maximum likelihood trees of the original sequence alignments using the phylogenetic tree summarization program SumTrees [80].

\section{Additional files}

Additional file 1: Additional tables. Includes genome assemblies used in this study (Table S1), CEP genes identified in those genomes (with symbolic names for CEP genes found in the model species $A$. thaliana, $M$. truncatula and O. sativa) and their properties (Table S2), and identified CEP domains with their bit scores and associated CEP genes (Table S3).

Additional file 2: MEME output for the canonical CEP domain. Includes the CEP domain sequence logo and position-specific probability matrix (PSPM), iteratively generated from previously identified CEP domain sequences.

Additional file 3: $\mathrm{d}_{\mathrm{N}} / \mathrm{d}_{\mathrm{S}}$ ratios for orthologous groups. Each table contains mean values and high probability density (HPD) intervals of $d_{N} / d_{s}$ ratios, across all residues of the CEP prepropeptide for each orthologous group.

Additional file 4: Viewable with FigTree [http://tree.bio.ed.ac.uk/ software/figtree/]. CEP gene tree generated using amino acid sequences, with bootstrap support values.

Additional file 5: Viewable with FigTree [http://tree.bio.ed.ac.uk/ software/figtree/]. CEP gene tree generated using nucleotide sequences, with bootstrap support values.

Additional file 6: Viewable with FigTree [http://tree.bio.ed.ac.uk/ software/figtree/]. Rooted phylogenetic tree of plants used to weight CEP genes identified by organism.

Additional file 7: Can be extracted using 7-Zip [http://www.7-zip. org/]. All Python, R and shell scripts used to analyze genomes and generate the results for this study.

\section{Competing interests}

$\mathrm{NI}$ and MAD have filed a patent (WO/2013/104026) on using CEP peptides to manipulate plant architecture and nutrient uptake. HAO has no competing interests.

\section{Authors' contributions}

$\mathrm{HAO}, \mathrm{NI}$ and MAD conceived the study, designed the bioinformatic analysis and drafted the manuscript. HAO implemented the analysis pipeline including writing all custom scripts. All authors read and approved the final manuscript.

\section{Acknowledgements}

We thank Jason Bragg for his input and advice on inferring gene trees. This work was supported by an Australian Research Council Discovery Project grant (DP120101893). HAO received financial support (UHS10488) to conduct this study from the Grains Research and Development Council. Maximum likelihood gene trees were reconstructed using a computer cluster managed by the Genome Discovery Unit of the Australian Cancer Research Foundation's Biomolecular Resource Facility.

Received: 27 June 2014 Accepted: 24 September 2014 Published: 6 October 2014

\section{References}

1. Czyzewicz N, Yue K, Beeckman T, Smet ID: Message in a bottle: small signalling peptide outputs during growth and development. J Exp Bot 2013, 64:5281-5296.
2. Delay C, Imin N, Djordjevic MA: Regulation of Arabidopsis root development by small signaling peptides. Front Plant Sci 2013, 4:352.

3. Matsubayashi Y: Post-translational modifications in secreted peptide hormones in plants. Plant Cell Physiol 2011, 52:5-13.

4. Matsubayashi Y: Recent progress in research on small post-translationally modified peptide signals in plants. Genes Cells 2012, 17:1-10.

5. Fernandez A, Drozdzecki A, Hoogewijs K, Nguyen A, Beeckman T, Madder A, Hilson P: Transcriptional and functional classification of the GOLVEN/ROOT GROWTH FACTOR/CLE-Like signaling peptides reveals their role in lateral root and hair formation. Plant Physio/ 2013, 161:954-970.

6. Delay C, Imin N, Djordjevic MA: CEP genes regulate root and shoot development in response to environmental cues and are specific to seed plants. J Exp Bot 2013, 64:5383-5394.

7. Imin N, Mohd-Radzman NA, Ogilvie HA, Djordjevic MA: The peptide encoding MtCEP1 gene modulates lateral root and nodule numbers in Medicago truncatula. J Exp Bot 2013, 64:5395-5409.

8. Okamoto S, Shinohara H, Mori T, Matsubayashi Y, Kawaguchi M: Root-derived CLE glycopeptides control nodulation by direct binding to HAR1 receptor kinase. Nat Commun 2013, 4:2191.

9. Roberts I, Smith S, Rybel BD, Broeke JVD, Smet W, Cokere SD, Mispelaere M, Smet ID, Beeckman T: The CEP family in land plants: evolutionary analyses, expression studies, and a role in Arabidopsis shoot development. J Exp Bot 2013, 64:5371-5381.

10. Okuda S, Tsutsui H, Shiina K, Sprunck S, Takeuchi H, Yui R, Kasahara RD, Hamamura Y, Mizukami A, Susaki D, Kawano N, Sakakibara T, Namiki S, Itoh K, Otsuka K, Matsuzaki M, Nozaki H, Kuroiwa T, Nakano A, Kanaoka MM, Dresselhaus T, Sasaki N, Higashiyama T: Defensin-like polypeptide LUREs are pollen tube attractants secreted from synergid cells. Nature 2009, 458:357-361.

11. Ohyama K, Ogawa M, Matsubayashi Y: Identification of a biologically active, small, secreted peptide in Arabidopsis by in silico gene screening, followed by LC-MS-based structure analysis. Plant J 2008, 55:152-160

12. Bobay BG, DiGennaro P, Scholl E, Imin N, Djordjevic MA, Mck Bird D: Solution NMR studies of the plant peptide hormone CEP inform function. FEBS Lett 2013, 587:3979-3985.

13. den Akker SE, Lilley CJ, Danchin EGJ, Rancurel C, Cock PJA, Urwin PE, Jones JT: The Transcriptome of Nacobbus aberrans Reveals Insights into the Evolution of Sedentary Endoparasitism in Plant-Parasitic Nematodes. Genome Biol Evol 2014, 6:2181-2194.

14. Zhou P, Silverstein KA, Gao L, Walton JD, Nallu S, Guhlin J, Young ND: Detecting small plant peptides using SPADA (Small Peptide Alignment Discovery Application). BMC Bioinformatics 2013, 14:335.

15. Rensing SA, Lang D, Zimmer AD, Terry A, Salamov A, Shapiro H, Nishiyama T, Perroud P-F, Lindquist EA, Kamisugi Y, Tanahashi T, Sakakibara K, Fujita T, Oishi K, Shin-I T, Kuroki Y, Toyoda A, Suzuki Y, Hashimoto S, Yamaguchi K, Sugano S, Kohara Y, Fujiyama A, Anterola A, Aoki S, Ashton N, Barbazuk WB, Barker E, Bennetzen JL, Blankenship R, et al: The Physcomitrella genome reveals evolutionary insights into the conquest of land by plants. Science 2008, 319:64-69.

16. Banks JA, Nishiyama T, Hasebe M, Bowman JL, Gribskov M, DePamphilis C, Albert VA, Aono N, Aoyama T, Ambrose BA, Ashton NW, Axtell MJ, Barker E, Barker MS, Bennetzen JL, Bonawitz ND, Chapple C, Cheng C, Correa LGG, Dacre M, DeBarry J, Dreyer I, Elias M, Engstrom EM, Estelle M, Feng L, Finet C, Floyd SK, Frommer WB, Fujita T, et al: The Selaginella genome identifies genetic changes associated with the evolution of vascular plants. Science 2011, 332:960-963.

17. Chamala S, Chanderbali AS, Der JP, Lan T, Walts B, Albert VA, DePamphilis CW, Leebens-Mack J, Rounsley S, Schuster SC, Wing RA, Xiao N, Moore R, Soltis PS, Soltis DE, Barbazuk WB: Assembly and validation of the genome of the nonmodel basal angiosperm amborella. Science 2013, 342:1516-1517.

18. Nystedt B, Street NR, Wetterbom A, Zuccolo A, Lin Y-C, Scofield DG, Vezzi F, Delhomme N, Giacomello S, Alexeyenko A, Vicedomini R, Sahlin K, Sherwood E, Elfstrand M, Gramzow L, Holmberg K, Hällman J, Keech O, Klasson L, Koriabine M, Kucukoglu M, Käller M, Luthman J, Lysholm F, Niittylä T, Olson Å, Rilakovic N, Ritland C, Rosselló JA, Sena J, et al: The Norway spruce genome sequence and conifer genome evolution. Nature 2013, 497:579-584. 
19. Neale DB, Wegrzyn JL, Stevens KA, Zimin AV, Puiu D, Crepeau MW, Cardeno C, Koriabine M, Holtz-Morris AE, Liechty JD, Martínez-García PJ, Vasquez-Gross HA, Lin BY, Zieve JJ, Dougherty WM, Fuentes-Soriano S, Wu L-S, Gilbert D, Marçais G, Roberts M, Holt C, Yandell M, Davis JM, Smith KE, Dean JF, Lorenz WW, Whetten RW, Sederoff R, Wheeler N, McGuire PE, et al: Decoding the massive genome of loblolly pine using haploid DNA and novel assembly strategies. Genome Biol 2014, 15:R59.

20. Kalanchoe - Cbcb. [https://wiki.umiacs.umd.edu/cbcb/index.php/ Kalanchoe]

21. Finet C, Timme RE, Delwiche CF, Marlétaz F: Multigene phylogeny of the green lineage reveals the origin and diversification of land plants. Curr Biol 2010, 20:2217-2222.

22. IUPAC-IUB Joint Commission on Biochemical Nomenclature (JCBN): Nomenclature and symbolism for amino acids and peptides. Eur $J$ Biochem 1984, 138:9-37.

23. Cheng S, van den Bergh E, Zeng P, Zhong X, Xu J, Liu X, Hofberger J, de Bruijn S, Bhide AS, Kuelahoglu C, Bian C, Chen J, Fan G, Kaufmann K, Hall JC, Becker A, Bräutigam A, Weber APM, Shi C, Zheng Z, Li W, Lv M, Tao Y, Wang J, Zou H, Quan Z, Hibberd JM, Zhang G, Zhu X-G, Xu X, et al: The Tarenaya hassleriana genome provides insight into reproductive trait and genome evolution of crucifers. Plant Cell 2013, 25:2813-2830.

24. Birol I, Raymond A, Jackman SD, Pleasance S, Coope R, Taylor GA, Yuen MMS, Keeling Cl, Brand D, Vandervalk BP, Kirk H, Pandoh P, Moore RA, Zhao Y, Mungall AJ, Jaquish B, Yanchuk A, Ritland C, Boyle B, Bousquet J, Ritland K, MacKay J, Bohlmann J, Jones SJM: Assembling the $20 \mathrm{~Gb}$ white spruce (Picea glauca) genome from whole-genome shotgun sequencing data. Bioinformatics 2013, 29:1492-1497.

25. Yang Z: Likelihood ratio tests for detecting positive selection and application to primate lysozyme evolution. Mol Biol Evol 1998, 15:568-573.

26. Brayer KJ, Segal DJ: Keep your fingers off my DNA: protein-protein interactions mediated by $\mathrm{C} 2 \mathrm{H} 2$ Zinc Finger domains. Cell Biochem Biophys 2008, 50:111-131.

27. Yaffe MB, Elia AEH: Phosphoserine/threonine-binding domains. Curr Opin Cell Biol 2001, 13:131-138.

28. Zhang G, Guo G, Hu X, Zhang Y, Li Q, Li R, Zhuang R, Lu Z, He Z, Fang X, Chen L, Tian W, Tao Y, Kristiansen K, Zhang X, Li S, Yang H, Wang J, Wang J: Deep RNA sequencing at single base-pair resolution reveals high complexity of the rice transcriptome. Genome Res 2010, 20:646-654.

29. Sakai $H$, Mizuno $H$, Kawahara $Y$, Wakimoto $H$, Ikawa $H$, Kawahigashi $H$, Kanamori H, Matsumoto T, Itoh T, Gaut BS: Retrogenes in rice (Oryza sativa L. ssp. japonica) exhibit correlated expression with their source genes. Genome Biol Evol 2011, 3:1357-1368.

30. Krishnan NM, Pattnaik $S$, Jain $P$, Gaur $P$, Choudhary R, Vaidyanathan $S$, Deepak S, Hariharan AK, Krishna PB, Nair J, Varghese L, Valivarthi NK, Dhas K, Ramaswamy K, Panda B: A draft of the genome and four transcriptomes of a medicinal and pesticidal angiosperm Azadirachta indica. BMC Genomics 2012, 13:464.

31. Oelkers K, Goffard N, Weiller GF, Gresshoff PM, Mathesius U, Frickey T: Bioinformatic analysis of the CLE signaling peptide family. BMC Plant Biol 2008, 8:1.

32. Kinoshita A, Nakamura Y, Sasaki E, Kyozuka J, Fukuda H, Sawa S: Gain-of-function phenotypes of chemically synthetic CLAVATA3/ESR-related (CLE) peptides in Arabidopsis thaliana and Oryza sativa. Plant Cell Physiol 2007, 48:1821-1825.

33. Miyawaki K, Tabata R, Sawa S: Evolutionarily conserved CLE peptide signaling in plant development, symbiosis, and parasitism. Curr Opin Plant Biol 2013, 16:598-606.

34. Mohd-Radzman NA, Djordjevic MA, Imin N: Nitrogen modulation of legume root architecture signaling pathways involves phytohormones and small regulatory molecules. Front Plant Sci 2013, 4:385.

35. Strabala TJ, Phillips L, West M, Stanbra L: Bioinformatic and phylogenetic analysis of the CLAVATA3/EMBRYO-SURROUNDING REGION (CLE) and the CLE-LIKE signal peptide genes in the Pinophyta. BMC Plant Biol 2014, $14: 47$

36. Strabala TJ, MacMillan CP: The Arabidopsis wood model-the case for the inflorescence stem. Plant Sci 2013, 210:193-205.

37. Serres-Giardi L, Belkhir K, David J, Glémin S: Patterns and evolution of nucleotide landscapes in seed plants. Plant Cell 2012, 24:1379-1397.

38. Tagliabracci VS, Engel JL, Wen J, Wiley SE, Worby CA, Kinch LN, Xiao J, Grishin NV, Dixon JE: Secreted kinase phosphorylates extracellular proteins that regulate biomineralization. Science 2012, 336:1150-1153.
39. Kiyohara S, Sawa S: CLE Signaling systems during plant development and nematode infection. Plant Cell Physiol 2012, 53:1989-1999.

40. Haerty W, Golding GB: Low-complexity sequences and single amino acid repeats: not just "junk" peptide sequences. Genome 2010, 53:753-762.

41. Bailey TL, Williams N, Misleh C, Li WW: MEME: discovering and analyzing DNA and protein sequence motifs. Nucleic Acids Res 2006 34(suppl 2):W369-W373.

42. Won H, Renner SS: Dating dispersal and radiation in the gymnosperm gnetum (Gnetales) - clock calibration when outgroup relationships are uncertain. Syst Biol 2006, 55:610-622.

43. Beilstein MA, Nagalingum NS, Clements MD, Manchester SR, Mathews S: Dated molecular phylogenies indicate a Miocene origin for Arabidopsis thaliana. Proc Natl Acad Sci 2010, 107:18724-18728.

44. Schaefer $H$, Heibl C, Renner SS: Gourds afloat: a dated phylogeny reveals an Asian origin of the gourd family (Cucurbitaceae) and numerous oversea dispersal events. Proc R Soc B Biol Sci 2009, 276:843-851.

45. Wurdack KJ, Hoffmann P, Chase MW: Molecular phylogenetic analysis of uniovulate Euphorbiaceae (Euphorbiaceae sensu stricto) using plastid RBCL and TRNL-F DNA sequences. Am J Bot 2005, 92:1397-1420.

46. Lavin M, Herendeen PS, Wojciechowski MF: Evolutionary rates analysis of leguminosae implicates a rapid diversification of lineages during the tertiary. Syst Biol 2005, 54:575-594.

47. Rousseau-Gueutin M, Gaston A, Ainouche A, Ainouche ML, Olbricht K Staudt G, Richard L, Denoyes-Rothan B: Tracking the evolutionary history of polyploidy in Fragaria L. (strawberry): New insights from phylogenetic analyses of low-copy nuclear genes. Mol Phylogenet Evol 2009, 51:515-530.

48. Schäferhoff B, Fleischmann A, Fischer E, Albach DC, Borsch T, Heubl G, Müller KF: Towards resolving Lamiales relationships: insights from rapidly evolving chloroplast sequences. BMC Evol Biol 2010, 10:352.

49. Xi Z, Ruhfel BR, Schaefer $H$, Amorim AM, Sugumaran M, Wurdack KJ, Endress PK, Matthews ML, Stevens PF, Mathews S, Davis CC: Phylogenomics and a posteriori data partitioning resolve the Cretaceous angiosperm radiation Malpighiales. Proc Natl Acad Sci 2012, 109:17519-17524.

50. Bombarely A, Rosli HG, Vrebalov J, Moffett P, Mueller LA, Martin GB: A draft genome sequence of Nicotiana benthamiana to enhance molecular plant-microbe biology research. Mol Plant Microbe Interact 2012, 25:1523-1530

51. Ammiraju JSS, Lu F, Sanyal A, Yu Y, Song X, Jiang N, Pontaroli AC, Rambo T, Currie J, Collura K, Talag J, Fan C, Goicoechea JL, Zuccolo A, Chen J, Bennetzen JL, Chen M, Jackson S, Wing RA: Dynamic evolution of oryza genomes is revealed by comparative genomic analysis of a genus-wide vertical data set. Plant Cell 2008, 20:3191-3209.

52. Grass Phylogeny Working Group II: New grass phylogeny resolves deep evolutionary relationships and discovers C4 origins. New Phytol 2012, 193:304-312.

53. Potter D, Eriksson T, Evans RC, Oh S, Smedmark JEE, Morgan DR, Kerr M, Robertson KR, Arsenault M, Dickinson TA, Campbell CS: Phylogeny and classification of Rosaceae. Plant Syst Evol 2007, 266:5-43.

54. Särkinen $T$, Bohs L, Olmstead RG, Knapp S: A phylogenetic framework for evolutionary study of the nightshades (Solanaceae): a dated 1000-tip tree. BMC Evol Biol 2013, 13:214.

55. Yamane K, Kawahara T: Intra- and interspecific phylogenetic relationships among diploid Triticum-Aegilops species (Poaceae) based on base-pair substitutions, indels, and microsatellites in chloroplast noncoding sequences. Am J Bot 2005, 92:1887-1898.

56. Tang H, Krishnakumar V, Bidwell S, Rosen B, Chan A, Zhou S, Gentzbittel L, Childs KL, Yandell M, Gundlach H, Mayer KF, Schwartz DC, Town CD: An improved genome release (version Mt4.0) for the model legume Medicago truncatula. BMC Genomics 2014, 15:312.

57. Young ND, Debellé F, Oldroyd GED, Geurts R, Cannon SB, Udvardi MK Benedito VA, Mayer KFX, Gouzy J, Schoof H, Van de Peer Y, Proost S, Cook DR, Meyers BC, Spannagl M, Cheung F, De Mita S, Krishnakumar V, Gundlach H, Zhou S, Mudge J, Bharti AK, Murray JD, Naoumkina MA, Rosen B, Silverstein KAT, Tang H, Rombauts S, Zhao PX, Zhou P, et al: The Medicago genome provides insight into the evolution of rhizobial symbioses. Nature 2011, 480:520-524.

58. Rice P, Longden I, Bleasby A: EMBOSS: the European molecular biology open software suite. Trends Genet 2000, 16:276-277.

59. Bailey TL, Boden M, Buske FA, Frith M, Grant CE, Clementi L, Ren J, Li WW, Noble WS: MEME Suite: tools for motif discovery and searching. Nucleic Acids Res 2009, 37(suppl 2):W202-W208. 
60. Cuesta S, Guzmán C, Alvarez JB: Allelic diversity and molecular characterization of puroindoline genes in five diploid species of the Aegilops genus. J Exp Bot 2013, 64:5133-5143.

61. Christensen AC, Lyznik A, Mohammed S, Elowsky CG, Elo A, Yule R, Mackenzie SA: Dual-domain, dual-targeting organellar protein presequences in arabidopsis can use Non-AUG start codons. Plant Cell 2005, 17:2805-2816.

62. Simpson GG, Laurie RE, Dijkwel PP, Quesada V, Stockwell PA, Dean C, Macknight RC: Noncanonical translation initiation of the arabidopsis flowering time and alternative polyadenylation regulator FCA. Plant Cell 2010, 22:3764-3777.

63. Wamboldt Y, Mohammed S, Elowsky C, Wittgren C, de Paula WBM, Mackenzie SA: Participation of leaky ribosome scanning in protein dual targeting by alternative translation initiation in higher plants. Plant Cell 2009, 21:157-167.

64. Petersen TN, Brunak S, von Heijne G, Nielsen H: SignalP 4.0: discriminating signal peptides from transmembrane regions. Nat Methods 2011, 8:785-786.

65. Yang Z: PAML 4: Phylogenetic analysis by maximum likelihood. Mol Biol Evol 2007, 24:1586-1591.

66. Jones DT, Taylor WR, Thornton JM: The rapid generation of mutation data matrices from protein sequences. Comput Appl Biosci CABIOS 1992, 8:275-282.

67. Katoh K, Standley DM: MAFFT multiple sequence alignment software version 7: improvements in performance and usability. Mol Biol Evol 2013, 30:772-780.

68. Wickham H: ggplot2: Elegant Graphics for Data Analysis. 1st edition. New York: Springer; 2009.

69. Rivera MC, Jain R, Moore JE, Lake JA: Genomic evidence for two functionally distinct gene classes. Proc Natl Acad Sci 1998, 95:6239-6244.

70. Camacho C, Coulouris G, Avagyan V, Ma N, Papadopoulos J, Bealer K, Madden TL: BLAST+: architecture and applications. BMC Bioinformatics 2009, 10:421.

71. Salichos L, Rokas A: Evaluating ortholog prediction algorithms in a yeast model clade. PLoS One 2011, 6:e18755.

72. Lemey P, Minin VN, Bielejec F, Pond SLK, Suchard MA: A counting renaissance: combining stochastic mapping and empirical Bayes to quickly detect amino acid sites under positive selection. Bioinformatics 2012, 28:3248-3256.

73. Capella-Gutiérrez S, Silla-Martínez JM, Gabaldón T: trimAl: a tool for automated alignment trimming in large-scale phylogenetic analyses. Bioinformatics 2009, 25:1972-1973.

74. Hasegawa M, Kishino H, Yano T: Dating of the human-ape splitting by a molecular clock of mitochondrial DNA. J Mol Evol 1985, 22:160-174.

75. SMALT - Wellcome Trust Sanger Institute. [http://www.sanger.ac.uk/ resources/software/smalt/]

76. Trapnell C, Williams BA, Pertea G, Mortazavi A, Kwan G, van Baren MJ, Salzberg SL, Wold BJ, Pachter L: Transcript assembly and quantification by RNA-Seq reveals unannotated transcripts and isoform switching during cell differentiation. Nat Biotechnol 2010, 28:511-515.

77. Salichos L, Stamatakis A, Rokas A: Novel information theory-based measures for quantifying incongruence among phylogenetic trees. Mol Biol Evol 2014, 31:1261-1271.

78. Stamatakis A, Aberer AJ: Novel Parallelization Schemes for Large-Scale Likelihood-based Phylogenetic Inference. In 27th Int Symp Parallel Distrib Process. ; 2013:1195-1204

79. Stamatakis A: Phylogenetic models of rate heterogeneity: a high performance computing perspective. In 20th Int Symp Parallel Distrib Process. 2006.

80. Sukumaran J, Holder MT: DendroPy: a Python library for phylogenetic computing. Bioinformatics 2010, 26:1569-1571.

doi:10.1186/1471-2164-15-870

Cite this article as: Ogilvie et al:: Diversification of the C-TERMINALLY

ENCODED PEPTIDE (CEP) gene family in angiosperms, and evolution of plant-family specific CEP genes. BMC Genomics 2014 15:870.

\section{Submit your next manuscript to BioMed Central and take full advantage of:}

- Convenient online submission

- Thorough peer review

- No space constraints or color figure charges

- Immediate publication on acceptance

- Inclusion in PubMed, CAS, Scopus and Google Scholar

- Research which is freely available for redistribution 